\title{
Wave Phenomena in a Stratified Complex Plasma
}

\author{
Michael Kretschmer, Tetyana Antonova, Sergey Zhdanov, and Markus Thoma
}

\begin{abstract}
PK-4 is a plasma facility that uses a de discharge inside a glass tube for experiments on complex (dusty) plasmas in microgravity onboard the International Space Station. It was launched in October 2014. During development, several models of PK-4 were built. The experiments described here took place in the engineering model of PK-4. During a test run, we injected particles with different diameters. They formed a stratified complex plasma where an instability generated waves inside the strata. The analysis of the video data is shown and the type and the cause of the instability are discussed here.
\end{abstract}

Index Terms-Dusty plasmas, fluid dynamics, glow discharge devices, plasma instability, plasma waves.

\section{INTRODUCTION}

$\mathbf{P}$ K-4 IS a plasma facility onboard the International Space Station (ISS) [1]. PK stands for (German) plasma crystal. With its launch in 2014, PK-4 continues the successful history of complex (dusty) plasma experiment setups onboard the ISS since 2001 (PKE-Nefedov, PK-3 Plus) [2]. But unlike the former facilities, PK-4 uses mainly a dc discharge to generate a plasma inside a glass tube. Microparticles injected into this plasma acquire a negative charge due to the higher mobility of the electrons. Through a (screened) Coulomb force the particles interact with each other and form structures that can be visualized by video cameras. Depending on the plasma parameters, the complex plasma can be set to any state of matter: solid (crystalline), liquid, or gaseous, having one big advantage: in the video images, the dynamics of the system can be observed on the basis of individual particles, which is hardly possible with atoms in real matter. Furthermore, the tubular design of PK-4 is ideal for the study of complex plasmas in the liquid state-a unique experimental access to fluid dynamics at kinetic level of individual particles.

\section{DESCRIPTION OF THE EXPERIMENT}

\section{A. Experimental Setup}

The main plasma chamber of PK-4 is a glass tube with an inner diameter of $3 \mathrm{~cm}$ and an overall length of $50 \mathrm{~cm}$,

Manuscript received July 31, 2015; revised November 5, 2015; accepted November 9, 2015. Date of publication December 17, 2015; date of current version April 8, 2016. This work was supported by the German Aerospace Center through the PK-4 Project under Grant 50 WM 1442. The work of S. Zhdanov was supported by the European Research Council (ERC) through the European Union's Seventh Framework Programme (FP7/2007-2013)/ERC under Grant 267499. (Corresponding author: Michael Kretschmer.)

M. Kretschmer and M. Thoma are with Justus Liebig University, Giessen 35392, Germany (e-mail: michael.kretschmer@exp1.physik. uni-giessen.de; markus.h.thoma@exp1.physik.uni-giessen.de).

T. Antonova is with the German Aerospace Center, Weßling 82234, Germany (e-mail: tetyana.antonova@dlr.de).

S. Zhdanov is with the Max Planck Institute for Extraterrestrial Physics, Garching bei München 85748, Germany (e-mail: zh@mpe.mpg.de)

Color versions of one or more of the figures in this paper are available online at http://ieeexplore.ieee.org.

Digital Object Identifier 10.1109/TPS.2015.2501831 of which $35 \mathrm{~cm}$ contains the main plasma (Fig. 1). The chamber can be filled with a noble gas at a pressure of up to 10 mbar with a maximum gas flow rate of $20 \mathrm{sccm}$. A high-voltage (hv) dc source with several kilovolts drives an adjustable constant current (up to $3 \mathrm{~mA}$ ) through the gas, thus generating a low (i.e., room)-temperature plasma with a low ionization rate of $10^{-7}-10^{-6}$. In addition, the polarity of the hv generator can be switched with up to a few kilohertz changing the electric field inside the discharge with a frequency well below the ion and electron plasma frequencies. The much heavier dust particles usually do not react on this polarity switching; they just feel the time-averaged resulting field.

The monodisperse microparticles are injected by six different particle dispensers (D1-6). By switching the polarity in dc mode or by setting a non-50\% duty cycle in the switching mode, we can control the motion of the particles in the plasma. Further means for particle manipulation are: two radio frequency coils (movable RF1 and fixed RF2), a ring electrode [electrical manipulation (EM)], a thermal manipulation ring on the outside of the chamber, and a $20-\mathrm{W}$ infrared laser beam (optical manipulation), depicted in blue in Fig. 1.

The particles are illuminated by a planar $150-\mathrm{mW}$ green laser beam [illumination laser (IL)], coming from the right in Fig. 1. Two monochrome charge-coupled device cameras (PO1, PO2) with a resolution of $1600 \mathrm{p} \times 1200 \mathrm{p}(\mathrm{each})$ at a frame rate of up to 35 frames/s (at full resolution) film the particles illuminated by the IL. The pixel resolution is $14.5 \mu \mathrm{m} / \mathrm{p}$. The video images are recorded on solid-state disks.

\section{B. Experimental Procedure}

We filled the chamber with 0.8 mbar of Neon and ignited a 1-mA dc plasma. Gas flow was turned off. Then we injected 3.43- and 1.28- $\mu \mathrm{m}$ spherical melamine-formaldehyde particles from dispensers D5 and D6, respectively, into the plasma. In the dc mode, the particles drifted toward the anode (left side) but were stopped at the RF1 electrode that was positioned at location 150 , as shown in Fig. 1, and operated with a power of $0.6 \mathrm{~W}$ at $81 \mathrm{MHz}$. There the particles were kept for several minutes, and then another injection of small particles was made to top up the particle cloud. As usual, under normal gravity conditions, the different particles form layers: the small particles hovering on top of the large particles, clearly separated by a sharp borderline.

When we switched the hv generator from dc to $50 \%$ duty cycle in polarity switching mode, the particle cloud slowly started drifting back to the right side where it was stopped again at the location of the EM electrode. This electrode 


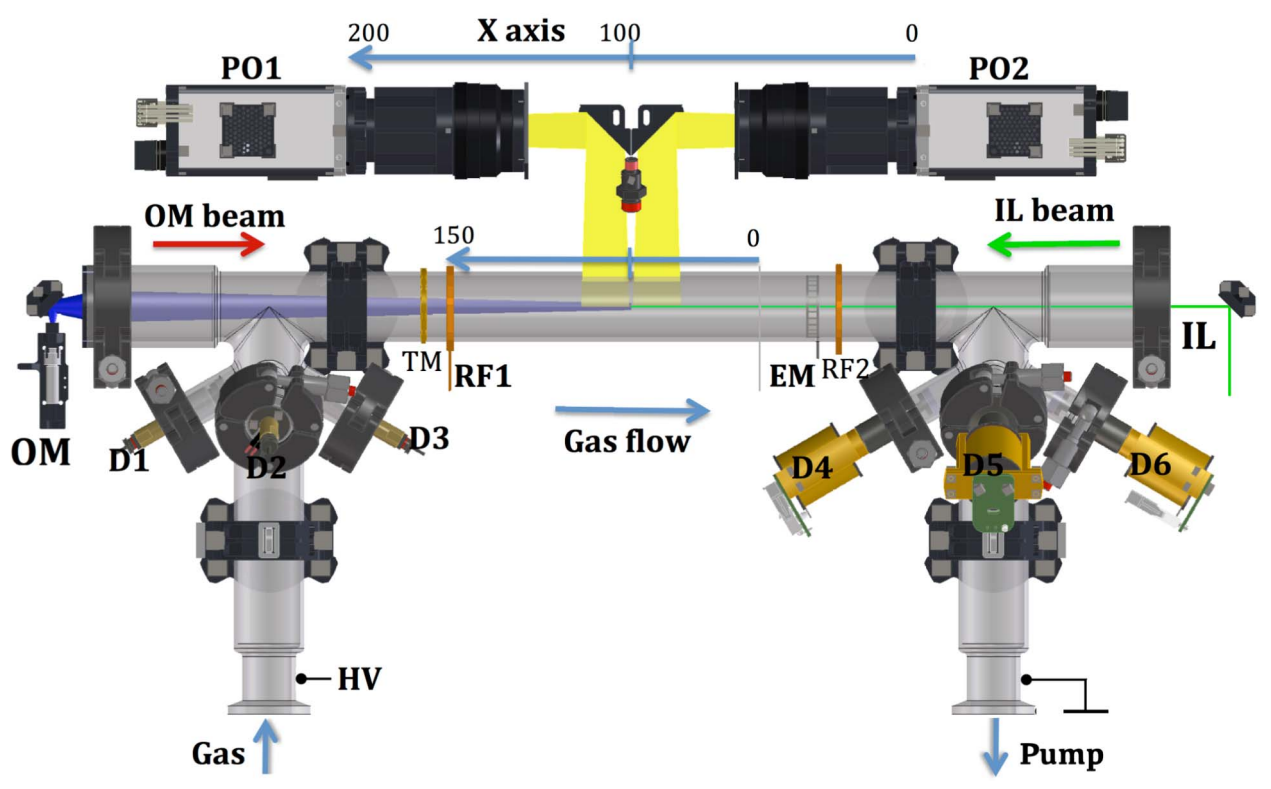

Fig. 1. Drawing of the plasma chamber of PK-4, a glass tube with an inner diameter of $3 \mathrm{~cm}$ and an overall length of $50 \mathrm{~cm}$, where $35 \mathrm{~cm}$ is filled with plasma captured by camera PO2. All the components used in the experiment can be found: particle dispensers (D5-6), IL, particle observation cameras (PO2), and particle manipulation devices (RF1, EM). The $x$-axis runs along the IL, that is from right to left. The same orientation is valid for Figs. 2 and 3.

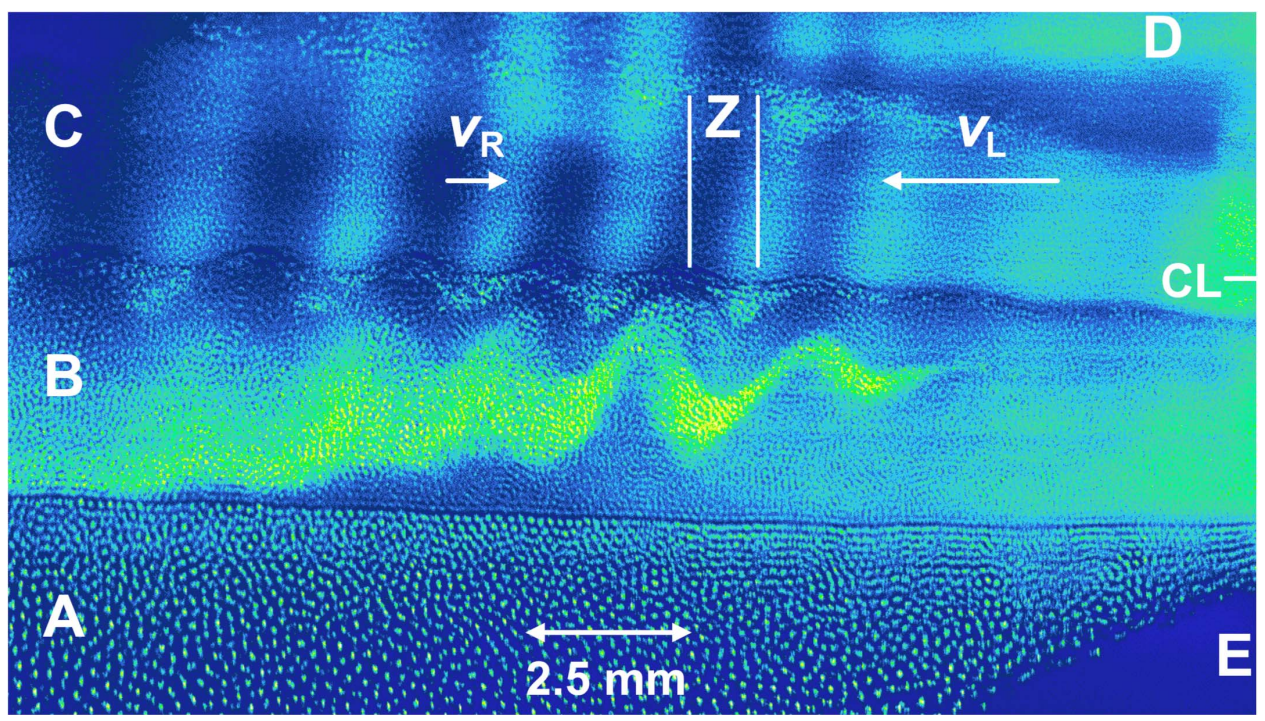

Fig. 2. Wave phenomena observed in a stratified complex plasma (false-color representation to enhance brightness variations). We can distinguish several layers of microparticles with different sizes: A, B, C, and D. E is the entry of a nozzle made up of electrode EM (Fig. 1) just outside of the image to the right. CL means central line of the tube. Density waves occur in layer C, propagating with velocities $v_{L}$ and $v_{R}$ in the directions depicted by the arrows. They merge in region $Z$. A transversal (ocean-like) wave can be seen in layer B. The image size is $18.6 \mathrm{~mm} \times 10.4 \mathrm{~mm}$.

consists of a metal band inside the glass tube. It was not actively used in our case, so it was electrically floating on a negative potential, thus repelling the microparticles and forming a kind of nozzle or barrier that finally stopped the particle cloud. In the final state, the cloud was stationary, but compressional waves emerging out of the nozzle and moving into the cloud were observed.

The events were recorded with a frame rate of 35 frames/s.

\section{OBSERVATIONS}

The scenario is shown in Fig. 2, that is one video image in false colors to emphasize the variation in pixel brightness.
We can clearly distinguish three main layers, A, B, and C. A contains the large particles (which collect a higher number of elementary charges visible in the larger interparticle distance). They mostly do not move, except for thermal motion, forming a stable ground that the other particles rest on.

In layer $\mathrm{B}$, we find the smaller and more recently injected particles. Finally, in layer $\mathrm{C}$ the first-injected small particles gather, also clearly separated from the B particles which have nominally the same size. But these particles have been captured in the RF1 discharge with a power of $0.6 \mathrm{~W}$ for several minutes, which led to a slight but noticeable reduction in their diameter due to sputtering by ions. 


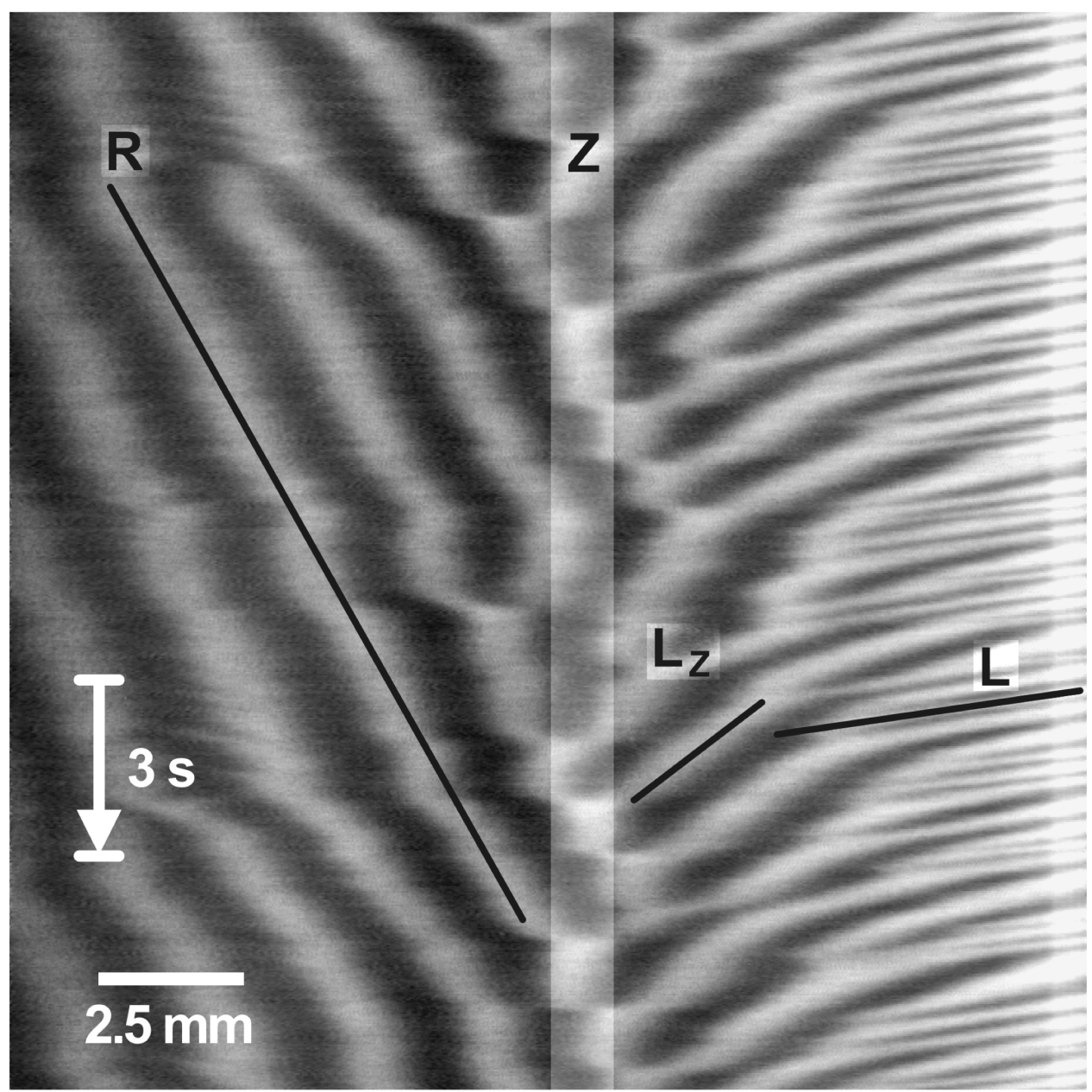

Fig. 3. Space-time diagram (periodgram) of the waves visible in Fig. 2, taken from a stripe along layer C. Time elapses from top to bottom. Scales are given: the arrow corresponds to a time of $3 \mathrm{~s}$ and the horizontal bar to $2.5 \mathrm{~mm}$. The wave velocities can be derived from the slopes of lines fitted to the wave maxima. Selected examples $\left(R, L, L_{Z}\right)$ for the calculation of $v_{R}, v_{L}$, and $v_{Z}$, respectively (see text), are shown.

Region D also contains a cloud of particles, but these particles are too small to be visible in the video image. These particles have not been injected. They grew from the material that has been sputtered away from the particles in C [3], another hint that sputtering must have really occurred in the RF discharge. These particles do not take part in the wave phenomenon, so they are no longer discussed here.

$\mathrm{E}$ in the lower right corner shows the entry of the nozzle produced by the floating EM electrode on the right-hand side, just outside of the image. CL depicts the geometrical centerline of the glass tube. The image shows a total size of $18.6 \mathrm{~mm} \times 10.4 \mathrm{~mm}$.

Some characteristic numbers of the layers A-C are summarized in Table I.

The plasma parameters are estimated according to [4]-[6] as follows: the electrical field strength along the tube $E=2 \mathrm{~V} / \mathrm{cm}$, the electron number density $n_{e}=3 \cdot 10^{8} \mathrm{~cm}^{-3}$, and the electron temperature $T_{e}=7 \mathrm{eV}$. This also provides the Debye screening lengths for electrons and ions with $\lambda_{\mathrm{De}}=1140 \mu \mathrm{m}$ and $\lambda_{\mathrm{Di}}=70 \mu \mathrm{m}$, respectively, and a mean free path of $\lambda_{\mathrm{ia}}=80 \mu \mathrm{m}$ for the ions.

Several species of waves are visible in the video [7] and in Fig. 2. In layer $\mathrm{C}$, very fast compressional waves emerge out
TABLE I

PROPERTIES OF THE PARTICLE LAYERS

\begin{tabular}{|c|c|c|r|c|c|}
\hline & $\begin{array}{c}\text { Height } \\
{[\mathrm{mm}]}\end{array}$ & $\begin{array}{c}\text { Part. Diam. } \\
{[\mu \mathrm{m}]}\end{array}$ & $\begin{array}{c}\text { Spacing } \\
{[\mu \mathrm{m}]}\end{array}$ & $\begin{array}{c}\text { Density } \\
{\left[10^{3} \mathrm{~cm}^{-3}\right]}\end{array}$ & $\begin{array}{c}\text { Charge } \\
{\left[\mathrm{e}^{-}\right]}\end{array}$ \\
\hline \multirow{2}{*}{$\mathrm{A}$} & $\begin{array}{c}\text { Not } \\
\text { visible }\end{array}$ & 3.43 & Top: 152 & 283 & 3100 \\
& Bottom: 237 & 58.6 & 4900 \\
\hline \multirow{2}{*}{$\mathrm{B}$} & $3.6(\mathrm{l})$ & \multirow{2}{*}{1.28} & Top: 128 & 479 & 1300 \\
& $3.0(\mathrm{r})$ & & Bottom: 112 & 717 & 1200 \\
\hline \multirow{2}{*}{$\mathrm{C}$} & $3.7(\mathrm{l})$ & $\leq 1.28$ & Dense: 85.0 & 1630 & \\
& $3.3(\mathrm{r})$ & & Rarefied: 113 & 685 & \\
\hline
\end{tabular}

Characteristics of the particle layers A-C from figure 2. Since the parameters vary inside one layer two numbers are given: Top and bottom in layers $\mathrm{A}$ and $\mathrm{B}$, and the numbers in the densified and rarefied phases of the compressional wave in layer $\mathrm{C}$. The height of each layer also changes from left (l) to right (r). The density is derived from the particle spacing. The particle charge is estimated by an extrapolation of probe measurements [4] and recommendations given in [6].

of the nozzle progressing from right to left with velocity $v_{L}$. On the left-hand side, there are also compressional waves in $\mathrm{C}$, but traveling much slower from left to right with $v_{R}$. In region $Z$, the waves combine and vanish without any superposition effects. The width of $Z$ is approximately half of the wavelength. 
TABLE II

WAVE CHARACTERISTICS

\begin{tabular}{|c|c|c|c|}
\hline & $\begin{array}{c}\text { Wavelength } \\
{[\mathrm{mm}]}\end{array}$ & $\begin{array}{c}\text { Frequency } \\
{[\mathrm{Hz}]}\end{array}$ & $\begin{array}{c}\text { Velocity } \\
{[\mathrm{mm} / \mathrm{s}]}\end{array}$ \\
\hline $\mathrm{A}$ & No waves & - & - \\
\hline B & Same as C & & \\
\hline C & R: 2.50 & 0.23 & $-0.57 \pm 0.05$ \\
& L: 2.57 & 2.69 & $6.54 \pm 0.73$ \\
\hline
\end{tabular}

Wavelength and frequency are estimated from the video data. The velocity is derived from the periodgram, figure 3 .

Especially, the slow waves on the left-hand side induce a transversal modulation of the boundary between $\mathrm{B}$ and $\mathrm{C}$ with the compressed phases pushing down the boundary and the rarefied phases releasing it up. The most prominent feature is visible in layer B: a transversal wave (yellow-green) that resembles a kind of surface wave, like a water wave on the ocean. It moves from left to right in phase with the compressional wave above and also exhibits from time to time breaking wave crests (e.g., visible on the second crest from the right side in Fig. 2), just like the well-known waves on a beach.

\section{ANALYSIS}

From Fig. 2 and the video images, we can derive some numbers to characterize the wave phenomena. A summary for layers $\mathrm{A}-\mathrm{C}$ is given in Table II.

To visualize the dynamics of the waves in a single image, a space-time diagram (periodgram) was created [8]. For this, a rectangle of 16 pixels height from left to right was extracted from layer $\mathrm{C}$ in each video frame. The pixel intensity $(0-255)$ was averaged over the height, resulting in one line representing the particle density inside the layer. Drawing these lines below each other gives us Fig. 3. The frame number (time) elapses from top to bottom. There we can see the waves travelling from left to right $(R)$, from right to left $(L)$, and merging in region $Z$. In a region next to $Z$ to the $\operatorname{right}\left(L_{Z}\right)$, we notice that the $L$ waves slow down before entering $Z$. By fitting lines to the maxima, we can derive velocities by measuring the slopes of the lines. After averaging, we find $-0.57 \pm 0.05 \mathrm{~mm} / \mathrm{s}$ for $v_{R}$ and $6.54 \pm 0.73 \mathrm{~mm} / \mathrm{s}$ for $v_{L}$ (with positive sign along the $x$-axis, i.e., from right to left), and a slowing down of $v_{L}$ to $1.28 \mathrm{~mm} / \mathrm{s}$ in the region $L_{Z}$.

If we generalize the velocity $\boldsymbol{v}_{x}=\boldsymbol{v}_{D}+\boldsymbol{C k} / k$, with $\boldsymbol{v}_{D}$ being an average velocity, $\boldsymbol{C}$ the dust sound speed, $k$ the wave number, and $\boldsymbol{k} / \boldsymbol{k}$ the wave propagation direction (positive in $+x$ direction), we can write

$$
v_{L}=6.54 \mathrm{~mm} / \mathrm{s}=v_{D}+C \text { (along } x \text { axis) }
$$

and

$$
v_{R}=-0.57 \mathrm{~mm} / \mathrm{s}=v_{D}-C \text { (against } x \text { axis). }
$$

This yields the velocities $C=3.56 \mathrm{~mm} / \mathrm{s}$ and $v_{D}=$ $2.99 \mathrm{~mm} / \mathrm{s}$, which are reasonable values in complex plasmas.

\section{DisCusSION}

\section{A. Instabilities}

The transversal wave in region B (the ocean wave) looks like a typical Kelvin-Helmholtz $(\mathrm{KH})$ instability. If we estimate the Epstein drag $D_{E}$ for $1.28 \mu \mathrm{m}$ particles in $0.8 \mathrm{mbar}$ of Neon gas, we find $D_{E}=490 \mathrm{~s}^{-1}$. This is much too high for KH, as shown in [9]. If we assume a Deep-Water Rayleigh-Taylor instability [10], [11] we find good agreement, since the spatial increment $[L$ in $\exp (x / L)]$ estimated from the first three maxima of the wave is found to be $L^{-1}=3-5 \mathrm{~cm}^{-1}$ (compared with $4 \mathrm{~cm}^{-1}$ in [9]).

\section{B. Cause of the Waves}

Instabilities in complex plasmas are a widely known phenomenon [12]-[19]. The cause of these (linear and non-linear) waves and vibrations are mostly space-dependent changes in plasma parameters or effects of streaming (sub or super-sonic) ions. In our case, we assume that the cause of the instability is related to the nozzle effect at the floating EM electrode. It can be speculated that the plasma parameters change within the nozzle or the velocity of the ions changes significantly due to the negative EM electrode, thus leading to the generation of the waves observed.

The appearance of the transversal (ocean) wave in region B is still under discussion. The particles inside the wave are much brighter than others (visible by the yellow and green color in Fig. 2). Usually, the brighter the particles are, the larger they are. But this is not the case here because larger particles would sink down to layer A. So this needs some more investigation.

\section{CONCLUSiON AND OUTLOOK}

An instability has been observed inside a stratified complex plasma under laboratory conditions with the PK-4 plasma facility. We saw compressional waves propagating in opposite direction with different velocities, inducing a transversal wave in one of the layers. This ocean wave could be characterized as a Deep-Water Rayleigh-Taylor instability.

Some of the observations need a deeper investigation. The experiment was repeated several times. It is planned to perform more experiments with different parameter settings, e.g., another current or frequency of polarity switching, to see whether this influences the behavior of the observed waves. These experiments have to be done again in a ground-based model of PK-4, not with the unit on the ISS, since this kind of particle stratification occurs usually only under normal gravity conditions.

\section{REFERENCES}

[1] M. H. Thoma et al., "PK-4: Complex plasmas in space-The next generation," IEEE Trans. Plasma Sci., vol. 35, no. 2, pp. 255-259, Apr. 2007.

[2] A. P. Nefedov et al., "PKE-Nefedov: Plasma crystal experiments on the international space station," New J. Phys., vol. 5, p. 33 , Apr. 2003.

[3] L. Wörner, "Tuning the interaction potential in complex plasmas," Ph.D. dissertation, Fakultät Phys., Ludwig-Maximilians-Univ. München, Munich, Germany, Oct. 2012. 
[4] A. Usachev et al., "The project 'Plasmakristall-4' (PK-4)—A dusty plasma experiment in a combined dc/rf(i) discharge plasma under microgravity conditions," Czechoslovak J. Phys., vol. 54, pp. C639-C647, Mar. 2004.

[5] G. E. Morfill et al., "The 'classical tunnelling effect'-Observations and theory," New J. Phys., vol. 8, p. 7, Jan. 2006.

[6] A. D. Usachev et al., "Formation of a boundary-free dust cluster in a low-pressure gas-discharge plasma," Phys. Rev. Lett., vol. 102, p. 045001, Jan. 2009

[7] Experiment Video. [Online]. Available: http://www.meikeltroniks.de/pub/ waves.html, accessed Nov. 10, 2015.

[8] M. Schwabe, M. Rubin-Zuzic, S. Zhdanov, H. M. Thomas, and G. E. Morfill, "Highly resolved self-excited density waves in a complex plasma," Phys. Rev. Lett., vol. 99, p. 095002, Aug. 2007.

[9] R. Heidemann, S. Zhdanov, K. R. Sütterlin, H. M. Thomas, and G. E. Morfill, "Shear flow instability at the interface among two streams of a highly dissipative complex plasma," Europhys. Lett., vol. 96, no. 1, p. 15001, 2011.

[10] J. W. Strutt and L. Rayleigh, "Investigation of the character of the equilibrium of an incompressible heavy fluid of variable density," Proc. London Math. Soc., vol. 14, pp. 170-177, Apr. 1883.

[11] G. Taylor, "The instability of liquid surfaces when accelerated in a direction perpendicular to their planes," Proc. R. Soc. Lond. A, Math. Phys. Sci., vol. 201, pp. 192-196, Dec. 1949.

[12] J. H. Chu, J.-B. Du, and L. I, "Coulomb solids and low-frequency fluctuations in RF dusty plasmas," J. Phys. D, Appl. Phys., vol. 27, no. 2, p. 296, 1994
[13] A. Barkan, R. L. Merlino, and N. D'Angelo, "Laboratory observation of the dust-acoustic wave mode," Phys. Plasmas, vol. 2, p. 3563, Oct. 1995 .

[14] M. Rosenberg and G. Kalman, "Dust acoustic waves in strongly coupled dusty plasmas," Phys. Rev. E, vol. 56, pp. 7166-7173, Dec. 1997.

[15] S. Khrapak et al., "Compressional waves in complex (dusty) plasmas under microgravity conditions," Phys. Plasmas, vol. 10, no. 1, pp. 1-4, 2003.

[16] M. Kretschmer et al., "Force field inside the void in complex plasmas under microgravity conditions," Phys. Rev. E, vol. 71, p. 056401, May 2005.

[17] J. D. Williams, E. Thomas, Jr., and L. Marcus, "Observations of vertically propagating driven dust acoustic waves: Finite temperature effects," Phys. Plasmas, vol. 15, no. 4, p. 043704, 2008.

[18] V. Nosenko, S. K. Zhdanov, S.-H. Kim, J. Heinrich, R. L. Merlino, and G. E. Morfill, "Measurements of the power spectrum and dispersion relation of self-excited dust acoustic waves," Europhys. Lett., vol. 88, no. 6, p. 65001, 2009.

[19] S. Zhdanov et al., "Dissipative dark solitons in a dc complex plasma," Europhys. Lett., vol. 89, p. 25001, Feb. 2010.

Authors' photographs and biographies not available at the time of publication. 